\title{
Child Psychiatry Branch of the National Institute of Mental Health Longitudinal Structural Magnetic Resonance Imaging Study of Human Brain Development
}

\author{
Jay N Giedd ${ }^{*, 1}$, Armin Raznahan ${ }^{1}$, Aaron Alexander-Bloch ${ }^{1}$, Eric Schmitt ${ }^{1}$, Nitin Gogtay ${ }^{1}$ and \\ Judith L Rapoport ${ }^{1}$ \\ ${ }^{1}$ Child Psychiatry Branch, NIMH, National Institutes of Mental Health, Bethesda, MD, USA
}

\begin{abstract}
The advent of magnetic resonance imaging, which safely allows in vivo quantification of anatomical and physiological features of the brain, has revolutionized pediatric neuroscience. Longitudinal studies are useful for the characterization of developmental trajectories (ie, changes in imaging measures by age). Developmental trajectories (as opposed to static measures) have proven to have greater power in discriminating healthy from clinical groups and in predicting cognitive/ behavioral measures, such as IQ. Here we summarize results from an ongoing longitudinal pediatric neuroimaging study that has been conducted at the Child Psychiatry Branch of the National Institute of Mental Health since 1989. Developmental trajectories of structural MRI brain measures from healthy youth are compared and contrasted with trajectories in attentiondeficit/hyperactivity disorder (ADHD) and childhood-onset schizophrenia. Across ages 5-25 years, in both healthy and clinical populations, white matter volumes increase and gray matter volumes follow an inverted $U$ trajectory, with peak size occurring at different times in different regions. At a group level, differences related to psychopathology are seen for gray and white matter volumes, rates of change, and for interconnectedness among disparate brain regions.

Neuropsychopharmacology Reviews (2015) 40, 43-49; doi: 10.1038/npp.20I4.236; published online 22 October 2014
\end{abstract}

\section{INTRODUCTION}

Magnetic resonance imaging (MRI) provides accurate measures of brain anatomy and physiology without the use of ionizing radiation. The safety of MRI has allowed not only the scanning of healthy children but of repeated scans throughout the course of development, generating data that have revolutionized the field of developmental neuroscience. Although MRI was developed in the 1940s, it was not used for pediatric neuroimaging studies of typical development until the 1980s. Technological improvements and wide spread availability of MRI scanners has led to a steadily increasing number of pediatric neuroimaging investigations and several conceptual progressions regarding analysis and interpretation.

Early studies targeted detection of gross pathology and non-specific findings, such as enlarged ventricles. The next generation of studies went beyond detection of gross pathology to addressing variation in the size of large regions

${ }^{*}$ Correspondence: Dr JN Giedd, Child Psychiatry Branch, NIMH, National Institutes of Mental Health, 10 Center Drive, MSC 1367, Building 10, Room 4C110, Bethesda, MD 20892, USA, Tel: +1 301435 4517, Fax: +1 301480 8898, E-mail: jg@nih.gov

Received 1 May 2014; revised 14 August 2014; accepted 22 August 2014; accepted article preview online 8 September 2014 of the brain (eg, frontal, temporal, parietal, and occipital lobes) and easily quantifiable components, such as the midsagittal area of the corpus callosum. The general trend of technological advances allowing for increasingly finer subdivisions of the brain continues with high-strength magnets capable of discerning individual layers of the cerebral cortex. However, most studies use magnets of 1.5 or 3.0 Tesla, with resolution of voxels approximately $1 \mathrm{~mm}^{3}$. Ever improving image analysis software, such as FreeSurfer, has become the main stay for most morphometric assessments although hand tracing by experienced raters remains the gold standard for quantification of some structures (eg, amygdala, hippocampus).

Another progression, afforded by larger sample sizes and longitudinal data, was the use of developmental trajectories (ie, change in size over time) as the measure of interest. Compared with static measures, developmental trajectories often yield greater correlation with cognitive/behavioral traits and genotype and greater discernment of old from young, male from female, and healthy from ill populations. However, even when using developmental trajectories, large inter-individual variation and substantial overlap between groups precludes the routine use of MRI for the diagnosis of psychiatric disorders. Likewise, predicting cognitive/behavioral traits from an individual scan with enough precision 
to be useful for educational or judicial decisions remains elusive.

The most recent analytical approach to pediatric neuroimaging investigations has been the use of graph theoretical approaches to examine 'connectivity' among disparate brain regions. As mental functioning emanates from the coordinated activity of disparate regions comprising neural networks, this approach makes intuitive sense. Parameters to characterize network properties of brain connectivity are increasingly being used to distinguish healthy from ill populations and to predict cognitive abilities.

Addressing neurodevelopmental hypotheses of mental illness (ie, that psychiatric disorders are manifestations of temporally diagnostically specific deviations from the path of healthy development) relies upon an adequate characterization of healthy development. As with diagnostic utility, large inter-individual variation makes precise characterization of often non-linear developmental trajectories challenging-usually necessitating large numbers of subjects with images acquired at multiple time points.

In this report, we update and summarize results of developmental trajectories from our ongoing longitudinal neuroimaging study of brain development at the Child Psychiatry Branch of the National Institute of Mental Health. The study design, initiated by Markus Krusei, MD in 1989 , is for subjects to come to the NIH at approximately 2-year intervals for MRI scans, neuropsychological testing, and genetic analysis. As of August 2014, the data base consists of $>6000$ scans from over 2000 subjects, approximately half from clinical populations such as ADHD, autism, and childhood-onset schizophrenia (COS), and half from typically developing individuals, including $>800$ scans from twins. The novel normative data presented here represent extensions of previously published results and are derived from 1171 longitudinally acquired structural MRI brain scans from 618 typically developing males and females aged 5-25 years.

\section{MRI OF BRAIN ANATOMY IN TYPICAL PEDIATRIC DEVELOPMENT}

Brain maturation in humans is protracted longer than any other species. Different tissue types, brain structures, and neural circuits have distinct developmental trajectories undergoing dynamic changes throughout life. The general pattern of change during childhood and adolescence is an increase in white matter (WM) volumes and inverted U-shaped trajectories of both cortical and subcortical gray matter (GM) volumes, with peak sizes occurring at different times in different regions.

\section{TOTAL CEREBRAL VOLUME}

As seen in Figure 1, the size of the brain and its subcomponents are highly variable with as much as a two-fold difference even among healthy subjects of the same age.

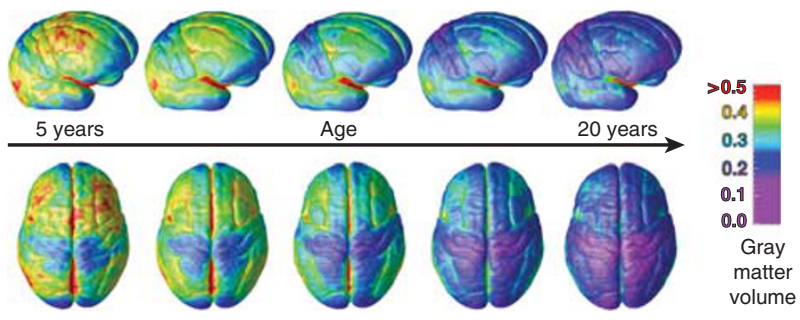

Figure 1. Cortical thinning during childhood and adolescence (Gogtay et al, 2004).

This high variability has important implications for the interpretation and utility of brain imaging results. Consistent with our prior report (Lenroot et al, 2007), total cerebral volume reaches $95 \%$ of its maximum size by age 6 years, increases slightly during childhood, reaching a peak at age 10.5 years in girls and 14.5 years in boys, and then subsequently slightly declines through the second and third decades (Figure 1a). Group-average total brain size is approximately $10 \%$ larger in males, and the magnitude of this difference is relatively stable from birth across the lifespan. This male/female difference in brain size should not be interpreted as imparting any sort of functional advantage or disadvantage as large-scale brain measures may not reflect sexually dimorphic differences in functionally relevant factors, such as neuronal connectivity and receptor density.

Across species there is a relationship between brain size and body size, but in individual humans the growth trajectories are quite dissimilar with body size increasing through approximately age 17 years. That sexual dimorphism of brain size is not solely attributable to the larger body size of males is especially evident in our pediatric population where brain size difference persists despite little difference in body size. In fact, both in our sample and according to published normative data from the Center for Disease Control, because of an earlier growth spurt, females tend to be slightly taller than males from ages 10-13 years.

Total brain size difference between males and females has broad implications for studies of sexual dimorphism. Many of the reported findings of brain sexual dimorphism are influenced strongly by whether, or how, the size of subcomponents of the brain are adjusted for the $\sim 10 \%$ difference in total brain volume. Without adjustment, the absolute size of most structures is larger in males. If adjustments are made to subcomponents (via covariation or the use of ratios to total brain volume), an entirely different list of structures, varying by sample size and age distribution, is generated.

Allometry, the study of the relationship between size and shape, is an important consideration in how best to account for male/female difference in subcomponents of the brain in light of the total size difference. The size of neurons, vasculature, and other components of brain anatomy are constrained by metabolic and physical properties necessitating brains of different sizes to have variable enlargement of parts (Finlay and Darlington, 1995). For instance, across 

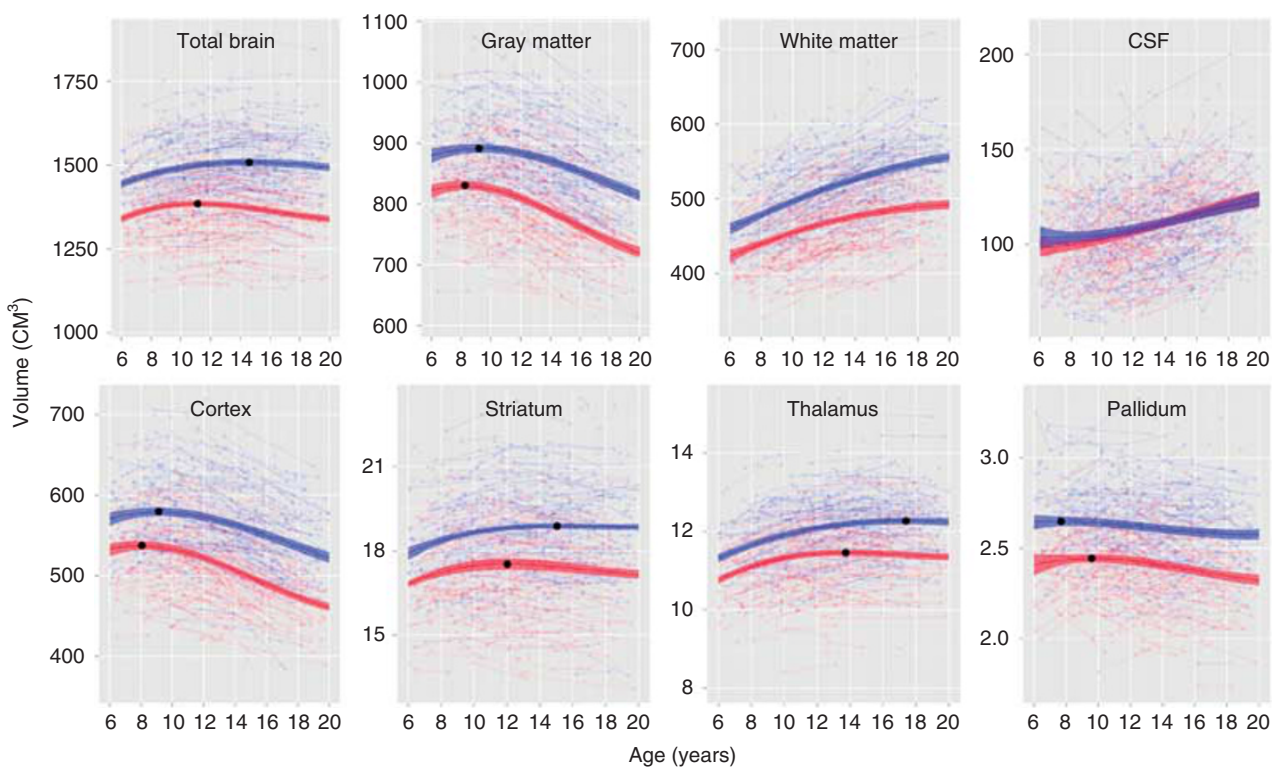

Figure 2. Raw data (faint) and fitted trajectories (bold) for structure volumes by sex (females/males), with volume peaks in age range $(\bullet)$.

and within species, WM-to-GM ratios increase with enlargement of total brain volume following a $4 / 3$ power law (Zhang and Sejnowski, 2000). This phenomenon may account for why reported differences of greater GM/WM ratios in females (Allen et al, 2003; Gur et al, 2002) are not nearly as robust when comparing males and females with similar total brain volumes (Leonard et al, 2008).

\section{GRAY MATTER}

GM is comprised predominantly of cell bodies and dendrites but may also include axons, glia, blood vessels, and extracellular space (Braitenberg, 2001). GM developmental trajectories follow an inverted U-shaped curve, with peak sizes occurring at different times and in different regions.

\section{Cortical}

Consistent with previous reports from a subset of the current sample, cortical GM volumes peak in the frontal lobes at 9.5 years in girls and 10.5 years in boys; in the temporal lobes at 10.0 years in girls and 11.0 years in boys; and in the parietal lobes at 7.5 years in girls and 9 years in boys. Figure 2 presents frames from a movie created from voxel level analysis of scans of 13 subjects who had each undergone scanning four times at approximately 2-year intervals between the ages of 4 and 22 years (movie available at http://www.nimh.nih.gov/videos/ press/prbrainmaturing.mpeg.) (Gogtay et al, 2004). The findings indicate that GM peaks the earliest in primary sensorimotor areas and the latest in higher-order association areas that integrate those primary functions (ie, dorsolateral prefrontal cortex, inferior parietal, and superior temporal gyrus).

Postmortem studies suggest that GM changes may be related in part to synaptic proliferation and pruning
(Huttenlocher, 1994). A study combining MRI and EEG in 138 healthy subjects from ages 10 to 30 years (Whitford et al, 2006) also indirectly implicates a connection between GM volume reductions and synaptic pruning. Reductions in frontal and parietal GM mirrored similar reductions in EEG power (which reflects synaptic activity). Another influence on GM volume (as assessed by MRI) is that myelination may change classification of voxels along the interior cortical border. This reclassification from GM to WM would not necessarily entail changes in the number of synapses (Sowell et al, 2001). Understanding the degree to which these and other processes may be driving the GM changes has profound implications for interpreting the imaging results. Imaging of nonhuman primates with postmortem validation may help discern the cellular phenomenon underlying the MRI changes.

\section{Subcortical}

The striatum, pallidum, and thalamus are involved in mediating movement and diverse developmentally emergent higher cognitive functions, attention allocation, and affective control. Anomalies of these structures have been reported for almost all neuropsychiatric disorders that have been investigated by neuroimaging (Giedd et al, 2006).

In our sample, the striatum, pallidum, and thalamus, like cortical GM, follow an inverted U-shaped developmental trajectory (Raznahan et al, 2014). Striatum and thalamus volumes peak later than cortical volumes and show a relative delay in males. Shape analysis indicates striatal, pallidal, and thalamic regions linked to specific frontoparietal association cortices contract with age, whereas other subcortical territories expand. Each of these subcortical GM structures has age-sensitive regions of sexual 
dimorphism that may have relevance for sex-biased mental disorders emerging in youth.

\section{WHITE MATTER}

Despite a lifelong reciprocal relationship and shared components of neural circuits, GM and WM have distinctly different developmental trajectories (Figure $1 \mathrm{~b}$ and $\mathrm{c}$ ). In contrast to the inverted U-shaped developmental trajectories of GM, WM volumes increase about $1-2 \%$ per year throughout childhood and adolescence (Figure 1c). Also in contrast to GM, WM increases are roughly similar across the major lobes (ie, the frontal, temporal, and parietal lobes). However, for smaller regions, the growth rates can be quite dynamic with as much as a $50 \%$ change over a 2 -year period (Thompson et al, 2000). The corpus callosum, comprised of approximately 200 million mostly myelinated axons connecting homologous areas of the left and right cerebral hemispheres, is the most prominent WM structure. Its developmental trajectory reflects the general increases in total WM volume.

WM volume largely reflects the degree of myelination, the wrapping of oligodendrocytes around axons that acts as an electrical insulator and serves to increase the speed of neuronal signal transmission up to 100 fold. Additionally, the process perpetuates a type of signal conduction in which signals 'hop' between gaps (called nodes of Ranvier) of myelination. Ion pumps then need to reset the ion gradients only at the nodes of Ranvier instead of along the entire expanse of the axons resulting in up to a 30 -fold increase in the frequency with which a given neuron can transmit information. The combination of increased speed $(100 \times)$ and quicker recovery time $(30 \times)$ can yield a 3000 -fold increase in bandwidth (ie, the amount of information transmitted per second). Myelination does not simply maximize speed of transmission, it fine-tunes the timing and synchrony of neuronal firing patterns (Fields and Stevens-Graham, 2002). Myelin also influences neuronal plasticity by releasing molecular factors that inhibit axon sprouting and the creation of new synapses (Fields, 2008). These non-subtle impacts of myelin on the brain's ability to process information and adapt to the environment may underlie many of the cognitive abilities associated with our species.

Increasing WM volumes subserve greater coherence on fMRI, EEG, and MEG and underlie a pattern of increased connectivity among spatially disparate brain regions that is a hallmark of child and adolescent development. The quantitative changes in WM volume are accompanied by qualitative changes as well. Diffusion tensor imaging studies, which assess directionality of WM, indicate a developmental increase in the organization of WM. Regionally specific changes in WM organization correlate with improvements in language (Nagy et al, 2004), reading (Deutsch et al, 2005), response inhibition ability (Liston et al, 2006), and memory (Nagy et al, 2004). Characterizing the changing relationships among disparate brain components is one of the most active areas of neuroimaging research, utilizing graph theory to quantify such things as small world network properties of the brain (Hagmann et al, 2010).

\section{CEREBROSPINAL FLUID}

Cerebrospinal fluid (ie, ventricular) volumes increase about $2 \%$ per year during typical child and adolescent development (Figure 1d), which should be considered when interpreting the many reports of increased ventricular volumes in a broad range of neuropsychiatric conditions. Increasing lateral ventricle volume measures are an indirect assessment of loss of the tissue from neighboring structures.

\section{CEREBELLUM}

The cerebellum is only about $1 / 10$ of the brain's volume, yet contains $80 \%$ of its neurons (Herculano-Houzel \& Lent, 2005). The cerebellum had been conceptualized as being related to motor control, but it is now commonly accepted that it is also involved in emotional processing and other higher cognitive functions that mature throughout adolescence (Riva and Giorgi, 2000; Schmahmann, 2004).

Because quantification of the cerebellum and its subdivision is not yet automated, we do not have measures on the entire sample. Manual tracing of 183 scans from a subset of 25 males and 25 females that each had $\geqslant 3$ longitudinally acquired scans indicated that total cerebellum size peaks at 11.3 years in girls and 15.6 years in boys (Tiemeier et al, 2010). Total cerebellum is $10-13 \%$ larger in males depending on the age of comparison. The sexual dimorphism remained significant after covarying for total brain volume. The cerebellum is comprised of phylogenetically and ontogenetically diverse subregions each with distinct developmental trajectories. The phylogenetically older vermis, which is the predominant component of the cerebellar cortex in many mammals, does not change in size across this age period. The phylogenetically more recent cerebellar hemispheres are among the latest of any structure to reach peak size continuing to grow into the late teens. The cerebellum is also distinctive in being the least heritable of structures we have examined. The cerebellum is also frequently implicated in childhood onset disorders, such as autism and ADHD. These features make the cerebellum a prime target for pediatric neuroimaging investigations.

\section{MRI OF BRAIN ANATOMY IN ATYPICAL PEDIATRIC DEVELOPMENT}

\section{Attention-Deficit/Hyperactivity Disorder}

ADHD is diagnosed in $5-10 \%$ of US school-age children, making it the most common pediatric neuropsychiatric condition (Kessler et al, 2005). Cross-sectional anatomical imaging studies of ADHD implicate decreased volume of the frontal lobes (Castellanos et al, 2002), parietal lobes (Sowell et al, 2003), basal ganglia (Castellanos and Giedd, 1994), 
corpus callosum (Giedd et al, 1994), and cerebellum (Berquin et al, 1998). Imaging studies of brain physiology also support involvement of right frontal-basal ganglia circuitry, with a powerful modulatory influence from the cerebellum (see (Giedd et al, 2001) for a review). Longitudinal studies of ADHD show a developmental delay of cortical thickness trajectories, most strikingly for the frontal regions (Shaw et al, 2007a). The most discriminating region was the middle prefrontal cortex where the median age at which $50 \%$ of the voxels reaching peak thickness was 10.9 years in those with ADHD and 5.9 years for controls.

In general, clinical improvement in ADHD is associated with a convergence of developmental trajectories towards typical development, whereas persistence of ADHD is associated with a progressive divergence away from typical development. For instance, right parietal cortical normalization accompanied clinical improvement (Shaw et al, 2006), and progressive volume loss of the inferior posterior lobes of the cerebellum accompanied persistence of ADHD (Mackie et $a l, 2007)$. Likewise, persistent $\mathrm{ADHD}$ is linked to a progressive loss of hippocampal volume (Shaw et al, 2007b). Stimulant drug treatment is associated with normalization of regional trajectories (Shaw et al, 2009).

Epidemiological and neuropsychological evidence indicates that ADHD can be viewed as the extreme of a continuous distribution of symptoms. This was illustrated by a study demonstrating that the ADHD pattern of slower rate of cortical thinning during late childhood and adolescence is linked with severity of symptoms of hyperactivity and impulsivity in typically developing children.

\section{CHILDHOOD-ONSET SCHIZOPHRENIA}

COS is defined by onset of psychotic symptoms before age 13 years. Although rare, occurring $\sim 1 / 500$ th as often as adult-onset schizophrenia (AOS), the COS cases ( $n=102$ to date) demonstrate neurobiological and phenomenological continuity with severe AOS (Gogtay and Rapoport, 2008). Similar to findings reported for adults with schizophrenia, COS subjects have enlarged ventricles and smaller volumes of total regional cortical GM, hippocampus, and amygdala. Basal ganglia volumes are larger and become more so during adolescence (see (Gogtay and Rapoport, 2008) for a review). Longitudinal data show delayed WM development (Gogtay et al, 2008) and an accelerated cortical GM loss during adolescence (Thompson et al, 2001). The degree of cortical thickness difference is attenuated by the early 20 s as control trajectory of cortical thinning 'catches up' with the accelerated pattern of cortical GM loss seen in COS. The cortical GM reduction in schizophrenia is related to loss of 'neuropil', which consists of glia, synaptic, and dendritic arbors and vasculature (Selemon and Goldman-Rakic, 1999). Postmortem studies do not show evidence of a glial response to neuronal injury or substantial neuronal loss suggested developmental models of abnormal synaptic function or structure (Weinberger et al, 1992).

\section{SUMMARY}

Specific developmental disorders have specific group anatomical brain differences. Although not of individual diagnostic utility, the imaging data are beginning to elucidate the timing and nature of deviations from typical development that may suggest therapeutic targets. Using trajectories (ie, morphometric measures by age) as an endophenotype may provide discriminating power where static measure do not (Giedd et al, 2008). It is increasingly clear that the same molecular genetic risk can be associated with a range of psychiatric phenotype, including autism, bipolar disorder, schizophrenia, mental retardation, and epilepsy. Conversely, the same psychiatric phenotype is likely to reflect numerous individually rare genetic abnormalities, such as copy number variants (Bassett et al, 2010; McClellan and King, 2010). Exploring the role of genetic variants on the timing of brain development may clarify some of these issues of sensitivity and specificity.

\section{FUTURE DIRECTIONS}

In addition to increasing utilization of graph theoretical approaches to examine neural circuitry across multiple modalities, future directions of neuroimaging will likely include seeking progress in the following four inter-related themes:

\section{Mapping $\rightarrow$ Mechanisms}

Neuroimaging studies are increasingly moving from simply mapping developmental trajectories to discerning mechanisms and influences. Comparing monozygotic (MZ) twins, who share $\sim 100 \%$ of the same genes, and dizygotic (DZ) twins, who share $\sim 50 \%$ of the same genes, is one approach to examine the relative contributions of genetic and environment interactions. Regionally specific changing heritability with age (Schmitt et al, 2014) highlights the importance of considering age $\times$ gene interactions. Agerelated changes in heritability may be related to the timing of gene expression and linked to the age of onset of disorders. There is a general trend for heritability to increase with age for WM and decrease for GM volumes (Lenroot et al, 2009; Wallace et al, 2006). The degree to which the same genetic or environmental factors contribute to multiple neuroanatomic structures can be assessed with multivariate analyses. In a pediatric sample of 326 twins and 158 singletons, shared effects account for more of the variance than structure-specific effects, with a single genetic factor accounting for $60 \%$ of variability in cortical thickness and five groups of structures strongly influenced by the same underlying genetic factors (Schmitt et al, 2007). Knowledge of when neural circuits are particularly sensitive to genetic or environmental influences could have important educational and/or therapeutic implications.

Male/female differences provide another opportunity to discern mechanisms of developmental change. Nearly all neuropsychiatric disorders have different prevalence, age of 
onset, and symptomatology between males and females. For instance, Autism is 4-7 times more common in males (the more severe the greater the discrepancy), whereas eating disorders are 9 times more common in females. In general, early onset disorders are more common in males (eg, autism, ADHD, dyslexia), whereas illness more common in females (eating disorders, anxiety, depression) become so only after puberty. Understanding the relative contributions of sex chromosomes and hormones may provide clues as to the mechanisms of development in health and illness (Clark et al, 1988; Diener et al, 1985; Morse et al, 1986). Toward this goal our group is studying subjects with anomalous sex chromosome variations (eg, $\mathrm{XXY}, \mathrm{XXX}, \mathrm{XXXY}, \mathrm{XYY}$ ) (Giedd et al, 2007), as well as subjects with anomalous hormone levels (eg, congenital adrenal hyperplasia, familial male precocious puberty, and Cushing syndrome) (Merke et al, 2003; Merke et al, 2005).

\section{Macro $\rightarrow$ Micro}

Another goal of future neuroimaging investigations will be to gain a greater understanding of the microanatomical changes underlying the MRI measures. The particular composition of a given voxel depends on its location in the brain, but a standard $1 \mathrm{~mm}^{3}$ voxel contains on average $3 \mathrm{~km}$ of axons, 90000 neurons, 400 meters of dendrites, and 4500000 synapses. To bridge the gap between micro and macro changes, crossspecies investigations may be helpful. Comparing postmortem data from people who had scans shortly before death may also shed light on the micro-macro relationships. One unresolved question that has substantial implications for the interpretation of many neuroimaging studies is the degree to which GM thinning is accounted for by synaptic pruning $v s$ greater myelination at the GM/WM border.

\section{Group $\rightarrow$ Individual}

A preeminent challenge of neuroimaging is to go beyond identifying differences at a group level to being able to predict cognitive or behavioral features in an individual. High variability, even among those of the same age and gender, and substantial overlap of most measures for most groups being compared limits the diagnostic utility of psychiatric neuroimaging (Lange et al, 1997). For example, although group average anatomical MRI differences have been reported for all major psychiatric disorders, MRI is not currently indicated for the routine diagnosis of any. Much of the utility of neuroimaging - whether clinical, judicial, or educational-relies on the degree to which this challenge can be met.

\section{Papers $\rightarrow$ People}

Neuroimaging investigations account for a substantial portion of research funding and have generated a large number of peer-reviewed journal articles. However, in psychiatry the impact on clinical practice has been modest.
The ultimate aim of future directions is to use the knowledge and insights unveiled to improve the lives of youth and their families.

\section{FUNDING AND DISCLOSURE}

The authors declare no conflict of interest.

\section{REFERENCES}

Allen JS, Damasio H, Grabowski TJ, Bruss J, Zhang W (2003). Sexual dimorphism and asymmetries in the gray-white composition of the human cerebrum. Neuroimage 18: 880-894.

Bassett AS, Costain G, Alan Fung WL, Russell KJ, Pierce L, Kapadia R et al (2010). Clinically detectable copy number variations in a Canadian catchment population of schizophrenia. J Psychiatr Res 44: 1005-1009.

Berquin PC, Giedd JN, Jacobsen LK, Hamburger SD, Krain AL, Rapoport JL et al (1998). Cerebellum in attention-deficit hyperactivity disorder: a morphometric MRI study. Neurology 50: 1087-1093.

Braitenberg $V$ (2001). Brain size and number of neurons: an exercise in synthetic neuroanatomy. J Comput Neurosci 10: 71-77.

Castellanos FX, Lee PP, Sharp W, Jeffries NO, Greenstein DK, Clasen LS et al (2002). Developmental trajectories of brain volume abnormalities in children and adolescents with attention-deficit/hyperactivity disorder. JAMA 288: 1740-1748.

Castellanos FX, Giedd JN (1994). Quantitative morphology of the caudate nucleus in ADHD. Biol Psychiatry 35: 725.

Clark AS, MacLusky NJ, Goldman-Rakic PS (1988). Androgen binding and metabolism in the cerebral cortex of the deveoping rhesus monkey. Endocrinology 123: 932-940.

Deutsch GK, Dougherty RF, Bammer R, Siok WT, Gabrieli JD, Wandell B (2005). Children's reading performance is correlated with white matter structure measured by diffusion tensor imaging. Cortex 41: 354-363.

Diener E, Sandvik E, Larsen RF (1985). Age and sex effects for affect intensity. Dev Psychol 21: 542-546.

Fields RD (2008). White matter in learning, cognition and psychiatric disorders. Trends Neurosci 31: 361-370.

Fields RD, Stevens-Graham B (2002). New insights into neuron-glia communication. Science 298: 556-562.

Finlay BL, Darlington RB (1995). Linked regularities in the development and evolution of mammalian brains. Science 268: 1578-1584.

Giedd JN, Blumenthal J, Molloy E, Castellanos FX (2001). Brain imaging of attention deficit/hyperactivity disorder. Ann NY Acad Sci 931: 33-49.

Giedd JN, Castellanos FX, Casey BJ, Kozuch P, King AC, Hamburger SD et al (1994). Quantitative morphology of the corpus callosum in attention deficit hyperactivity disorder. Am J Psychiatry 151: 665-669.

Giedd JN, Clasen LS, Wallace GL, Lenroot RK, Lerch JP, Wells EM et al (2007). XXY (Klinefelter syndrome): a pediatric quantitative brain magnetic resonance imaging case-control study. Pediatrics 119: e232-e240.

Giedd JN, Lenroot RK, Shaw P, Lalonde F, Celano M, White S et al (2008). Trajectories of anatomic brain development as a phenotype. Novartis Found Symp 289: 101-112. discussion 112-118, 193-195.

Giedd JN, Shaw P, Wallace G, Gogtay N, Lenroot RK (2006). Anatomic brain imaging studies of normal and abnormal brain development in children and adolescents. In: Cicchetti D, Cohen DJ (eds). Developmental Psychopathology. 2nd edn.Vol. 2, John Wiley \& Sons: Hoboken, NJ, USA, pp 127-194.

Gogtay N, Giedd JN, Lusk L, Hayashi KM, Greenstein D, Vaituzis AC et al (2004). Dynamic mapping of human cortical development during childhood through early adulthood. Proc Natl Acad Sci USA 101: 8174-8179.

Gogtay N, Lu A, Leow AD, Klunder AD, Lee AD, Chavez A et al (2008). Threedimensional brain growth abnormalities in childhood-onset schizophrenia visualized by using tensor-based morphometry. Proc Natl Acad Sci USA 105: 15979-15984.

Gogtay N, Rapoport JL (2008). Childhood-onset schizophrenia: insights from neuroimaging studies. J Am Acad Child Adolesc Psychiatry 47: 1120-1124.

Gur RC, Gunning-Dixon F, Bilker WB, Gur RE (2002). Sex differences in temporolimbic and frontal brain volumes of healthy adults. Cereb Cortex 12: 998-1003.

Hagmann P, Sporns O, Madan N, Cammoun L, Pienaar R, Wedeen VJ et al (2010). White matter maturation reshapes structural connectivity in the late developing human brain. Proc Natl Acad Sci USA 107: 19067-19072.

Herculano-Houzel S, Lent R (2005). Isotropic fractionator: a simple, rapid method for the quantification of total cell and neuron numbers in the brain. J Neurosci 25: 2518-2521. 
Huttenlocher PR (1994). Synaptogenesis in human cerebral cortex. In: Dawson G, Fischer K (eds). Human Behavior and the Developing Brain. Guilford Press: New York, NY, USA, pp 137-152.

Jerslid AT (1963). The Psychology of Adolescence. 2nd edn. Macmillan Publishing Company: New York, NY, USA.

Kessler RC, Berglund P, Demler O, Jin R, Merikangas KR, Walters EE (2005). Lifetime prevalence and age-of-onset distributions of DSM-IV disorders in the National Comorbidity Survey Replication. Arch Gen Psychiatry 62: 593-602.

Lange N, Giedd JN, Castellanos FX, Vaituzis AC, Rapoport JL (1997). Variability of human brain structure size: ages 4-20 years. Psychiatry Res 74: 1-12.

Lenroot RK, Gogtay N, Greenstein DK, Wells EM, Wallace GL, Clasen LS et al (2007). Sexual dimorphism of brain developmental trajectories during childhood and adolescence. Neuroimage 36: 1065-1073.

Lenroot RK, Schmitt JE, Ordaz SJ, Wallace GL, Neale MC, Lerch JP et al (2009). Differences in genetic and environmental influences on the human cerebral cortex associated with development during childhood and adolescence. Hum Brain Mapp 30: 163-174.

Leonard CM, Towler S, Welcome S, Halderman LK, Otto R, Eckert MA et al (2008). Size matters: cerebral volume influences sex differences in neuroanatomy. Cereb Cortex 18: 2920-2931.

Liston C, Watts R, Tottenham N, Davidson MC, Niogi S, Ulug AM et al (2006). Frontostriatal microstructure modulates efficient recruitment of cognitive control. Cereb Cortex 16: 553-560.

Mackie S, Shaw P, Lenroot R, Pierson R, Greenstein DK, Nugent TF et al (2007). Cerebellar development and clinical outcome in attention deficit hyperactivity disorder. Am J Psychiatry 164: 647-655.

McClellan J, King MC (2010). Genetic heterogeneity in human disease. Cell 141: 210-217.

Merke DP, Fields JD, Keil MF, Vaituzis AC, Chrousos GP, Giedd JN (2003). Children with classic congenital adrenal hyperplasia have decreased amygdala volume: Potential prenatal and postnatal hormonal effects. J Clin Endocrinol Metab 88: 1760-1765.

Merke DP, Giedd JN, Keil MF, Mehlinger SL, Wiggs EA, Holzer S et al (2005). Children experience cognitive decline despite reversal of brain atrophy one year after resolution of Cushing syndrome. J Clin Endocrinol Metab 90: 2531-2536.

Morse JK, Scheff SW, DeKosky ST (1986). Gonadal steroids influence axonal sprouting in the hippocampal dentate gyrus: a sexually dimorphic response. Exp Neurol 94: 649-658.

Nagy Z, Westerberg H, Klingberg T (2004). Maturation of white matter is associated with the development of cognitive functions during childhood. J Cogn Neurosci 16: 1227-1233.

Raznahan A, Shaw PW, Lerch JP, Clasen LS, Greenstein D, Berman R et al (2014). Longitudinal four-dimensional mapping of subcortical anatomy in human development. Proc Natl Acad Sci USA 111: 1592-1597.

Riva D, Giorgi C (2000). The cerebellum contributes to higher functions during development: evidence from a series of children surgically treated for posterior fossa tumours. Brain 123(Pt 5): 1051-1061.

Schmahmann JD (2004). Disorders of the cerebellum: ataxia, dysmetria of thought, and the cerebellar cognitive affective syndrome. J Neuropsychiatry Clin Neurosci 16: 367-378.
Schmitt JE, Neale MC, Fassassi B, Perez J, Lenroot RK, Wells EM et al (2014). The dynamic role of genetics on cortical patterning during childhood and adolescence. Proc Natl Acad Sci USA 111: 6774-6779.

Schmitt JE, Wallace GL, Rosenthal MA, Molloy EA, Ordaz S, Lenroot R et al (2007). A multivariate analysis of neuroanatomic relationships in a genetically informative pediatric sample. Neuroimage 35: 70-82.

Selemon LD, Goldman-Rakic PS (1999). The reduced neuropil hypothesis: a circuit based model of schizophrenia. Biol Psychiatry 45: 17-25.

Shaw P, Eckstrand K, Sharp W, Blumenthal J, Lerch JP, Greenstein D et al (2007a). Attention-deficit/hyperactivity disorder is characterized by a delay in cortical maturation. Proc Natl Acad Sci USA 104: 19649-19654.

Shaw P, Gornick M, Lerch J, Addington A, Seal J, Greenstein D et al (2007b). Polymorphisms of the dopamine D4 receptor, clinical outcome, and cortical structure in attention-deficit/hyperactivity disorder. Arch Gen Psychiatry 64: 921-931.

Shaw P, Lerch J, Greenstein D, Sharp W, Clasen L, Evans A et al (2006). Longitudinal mapping of cortical thickness and clinical outcome in children and adolescents with attention-deficit/hyperactivity disorder. Arch Gen Psychiatry 63: 540-549.

Shaw P, Sharp WS, Morrison M, Eckstrand K, Greenstein DK, Clasen LS et al (2009). Psychostimulant treatment and the developing cortex in attention deficit hyperactivity disorder. Am J Psychiatry 166: 58-63.

Sowell ER, Thompson PM, Tessner KD, Toga AW (2001). Mapping continued brain growth and gray matter density reduction in dorsal frontal cortex: Inverse relationships during postadolescent brain maturation. J Neurosci 21: 88198829.

Sowell ER, Thompson PM, Welcome SE, Henkenius AL, Toga AW, Peterson BS (2003). Cortical abnormalities in children and adolescents with attention-deficit hyperactivity disorder. Lancet 362: 1699-1707.

Thompson PM, Giedd JN, Woods RP, MacDonald D, Evans AC, Toga AW (2000). Growth patterns in the developing brain detected by using continuum mechanical tensor maps. Nature 404: 190-193.

Thompson PM, Vidal C, Giedd JN, Gochman P, Blumenthal J, Nicolson R et al (2001). Mapping adolescent brain change reveals dynamic wave of accelerated gray matter loss in very early-onset schizophrenia. Proc Natl Acad Sci USA 98: 11650-11655.

Tiemeier H, Lenroot RK, Greenstein DK, Tran L, Pierson R, Giedd JN (2010). Cerebellum development during childhood and adolescence: a longitudinal morphometric MRI study. Neuroimage 49: 63-70.

Wallace GL, Schmitt JE, Lenroot R, Viding E, Ordaz S, Rosenthal MA et al (2006). A pediatric twin study of brain morphometry. J Child Psychol Psychiatry 47: 987-993.

Weinberger DR, Berman KF, Suddath R, Torrey EF (1992). Evidence of dysfunction of a prefrontal-limbic network in schizophrenia: a magnetic resonance imaging and regional cerebral blood flow study of discordant monozygotic twins. Am J Psychiatry 149: 890-897.

Whitford TJ, Rennie CJ, Grieve SM, Clark CR, Gordon E, Williams LM (2006). Brain maturation in adolescence: Concurrent changes in neuroanatomy and neurophysiology. Hum Brain Mapp 28: 228-237.

Zhang K, Sejnowski TJ (2000). A universal scaling law between gray matter and white matter of cerebral cortex. Proc Natl Acad Sci USA 97: 5621-5626. 